Investigations

\title{
A State of PSI and PSII Photochemistry of Sunflower Yellow- Green Plastome Mutant
}

\author{
${ }^{1}$ Maksim S. Makarenko, ${ }^{2}$ Nikolay V. Kozel, \\ ${ }^{1}$ Alexander V. Usatov, ${ }^{3}$ Oleg F. Gorbachenko and ${ }^{2}$ Natalia G. Averina \\ ${ }^{1}$ Department of Genetics, Southern Federal University, Rostov-on-Don, Russia \\ ${ }^{2}$ Institute of Biophysics and Cell Engineering, National Academy of Sciences of Belarus, Minsk, Belarus \\ ${ }^{3}$ Zhdanov Don Experiment Station, All Russia Research Institute of Oil Crops, pos. Oporny, Rostov region, Russia
}

Article history

Received: 27-10-2016

Revised: $24-12-2016$

Accepted: 28-12-2016

Corresponding Author:

Maksim S. Makarenko

Department of Genetics,

Southern Federal University,

Rostov-on-Don, Russia

Email: mcmakarenko@yandex.ru

\begin{abstract}
Chlorophyll deficient mutants are appropriate model objects for a large number of investigations in the field of photosynthesis. In this study, we identified a state of Photosystem I (PSI) and Photosystem II (PSII) photochemistry and light responses of the sunflower yellow-green mutant line - en:chlorina-7, wherein mutations in chloroplast genes have been previously localized. The conducted research revealed low content of photooxidizable PSI and an impaired quantum yield of PSII photochemistry in the en:chlorina-7 line as compared with a wild type. Disturbances of PSI and PSII in yellow-green plastome mutant line of sunflower (en:chlorina-7) are associated with mutations in $p s a A$ and $p s b B$, respectively.
\end{abstract}

Keywords: Yellow-Green Mutants, Sunflower Plastome Mutant, PSI and PSII Reduction, $p s a A, p s b B$

\section{Introduction}

Chlorophyll (Chl) deficient mutants are the classic model objects for exploring the molecular mechanisms of photosynthetic apparatus biogenesis as well as the chloroplast development on the whole (Rassadina et al., 2005; Wang et al., 2014). Most of such kind researches are carried out on mutants with yellow-green leaf color, so called «chlorina phenotype» (Li et al., 2013; Brestic et al., 2015). Chlorina mutants have been identified in many higher plants, such as Arabidopsis (Harper et al., 2004), rice (Chen et al., 2013), wheat (Li et al., 2013), barley (Preiss and Thornber, 1995), maize (Asakura et al., 2008), tea (Wang et al., 2014), sunflower (Markin et al., 2016) and others. The investigation of genes associated with mutant phenotype is an actual issue for genetics of photosynthesis.

Chlorophyll mutations are of two types - nuclear or extranuclear. Virtually all chlorina lines used in the studies have nuclear origin of mutant phenotype. However chlorophyll deficient mutants with extranuclear genetic origin are of particular interest. This is primarily because mutations in plastid or mitochondrial DNA could not directly influence on Chl biosynthesis and the decrease of Chl occurs by retrograde signaling pathways involvement. Therefore, extranuclear chlorophyll mutants are also an appropriate model for investigating the interactions between organelles and nuclear genomes.
In the Southern Federal University, there have been obtained series of chlorophyll mutant lines using chemical mutagenesis (Beletskii et al., 1969). In current study we decided to use the yellow-green plastome mutant lineen:chlorina-7. The en:chlorina-7 line has chlorophyll bdeficient phenotype and characterized with about $35 \%$ decrease of chlorophyll $(\mathrm{a}+\mathrm{b})$ content and approximate $20 \%$ carotenoid reduction (Usatov et al., 2004). Previous studies of the en:chlorina-7 have revealed extranuclear genetic origin of mutant phenotype (Usatov et al., 2004). Further investigation by Markin et al. (2016) has shown 7 SNP in plastid genome of the en:chlorine-7 line compared to the 3629 line, however only 3 SNP in genes $r p o B, \quad p s a A, \quad p s b B$ were producing amino acid substitutions (nonsynonymous substitutions). The latter were represented by following substitutions: serine for leucine in 138 position of $\beta$-subunit of RNA-polymerase $(r p o B)$, threonine for isoleucine in 528 position of photosystem I P700 chlorophyll a apoprotein A1 ( $p s a A)$ and histidine for tyrosine in 157 position of photosystem II CP47 reaction center protein ( $p s b B)$ (Markin et al., 2016).

Comparative analysis of the en:chlorine-7 and the wild type line mitochondrial genomes has revealed no associations with chlorophyll deficient phenotype. Therefore the most obvious reasons for mutant phenotype could be mutations in $r p o B, p s a A, p s b B$. However using the BLAST program (https://www.ncbi.nlm.nih.gov/blast/) we found more than 
20 species with a normal Chl content which had nucleotide sequences of $r p o B 100 \%$ similar to the en:chlorina-7 allelic variant of $r p o B$ gene. So the $r p o B$ polymorphism is unlikely to be associated with the chlorina phenotype and here an influence of $p s a A, p s b B$ mutations on the phenotype has been considered in this study.

Although a genetic nature of the en:chlorina-7 mutant line is clear, there is a lack of information about specific photosynthetic responses of mutant plants. It is also unclear that is a primary reason for Chl deficiencyan impaired synthesis of Reaction Centers (RCs) or a reduction of Light Harvesting Complexes (LHC) molecules. In this study, we compare the state of Photo System I (PSI) and photosystem II (PSII) photochemistry and light responses of the en:chlorina-7 mutant compared to a wild type.

\section{Materials and Methods}

\section{Plant Material}

The study was carried out on the domesticated sunflower (Helianthus annuus) line 3629 and the yellowgreen plastome mutant sunflower line en:chlorina-7, which was obtained by a mutagenesis induced with $\mathrm{N}-$ nitroso-N-methylurea from an original inbred line 3629 . A technique of the inducible mutagenesis has been described earlier (Usatov et al., 2004).

Sunflower plants were gown in regularly irrigated pots in growth chamber KBWF 720 (Binder, Germany). The growing conditions were following: the temperature $-26^{\circ} \mathrm{C}$, the humidity $-70 \%$ and dark/light cycles- $10 / 14 \mathrm{~h}$. When plants have reached 4 th leaf stage, we conducted the measurements, using the both leaves from a third leaves pair. Seven plants from each line were studied.

\section{Chlorophyll Fluorescence Measurements}

To determine the chlorophyll fluorescence induction parameters PAM-fluorometer Dual-PAM-100 (Heinz Walz, Germany) with a chlorophyll fluorescence unit and P700 dual wavelength $(830 / 875 \mathrm{~nm})$ unit was used. Saturation pulses $\left(10,000 \mu \mathrm{mol}\right.$ photons $\left.\mathrm{m}^{-2} \mathrm{~s}^{-1}\right)$ were applied for the measurements of chlorophyll fluorescence parameters so for the assessment of the P700 parameters. Prior to measurements, all plants were dark adapted in a dark box for $30 \mathrm{~min}$. After the determination of $F O, F m$ and $P m$, the $150 \mu \mathrm{mol}$ photons $\mathrm{m}^{-2} \mathrm{~s}^{-1}$ light intensity was used for starting processes of photosynthesis. When the stable state was reached, a rapid light curve was induced (light intensities 30, 37, $46,77,119,150,240,363,555$ and $849 \mu \mathrm{mol}$ photons $\mathrm{m}^{-2} \mathrm{~s}^{-1} ; 30 \mathrm{sec}$ at each light intensity) also a saturation pulse and a far-red pulse were used for $\mathrm{F}^{\prime} \mathrm{o}$ measurements after $30 \mathrm{sec}$ at each light intensity. Following chlorophyll fluorescence parameters were calculated: $F v / F m=(F m-F o) / F m, Y(I I)=\left(F^{\prime} m /\right.$ $\left.F^{\prime}\right) / F^{\prime} m, \mathrm{qP}=\left(F^{\prime} m-F\right) /\left(F^{\prime} m-F^{\prime} \mathrm{o}\right), \mathrm{qN}=(F m-$
$\left.F^{\prime} m\right) /\left(F m-F_{o}\right), \mathrm{NPQ}=\left(F m-F^{\prime} m\right) / F^{\prime} m($ Oxborough and Baker, 1997; Kramer et al., 2004). Fo and is a value of minimum fluorescence in the $30 \mathrm{~min}$ darkadapted state and $F^{\prime} \mathrm{o}$ is a calculated value of the minimum fluorescence value in the light-adapted state (Lysenko et al., 2014). $F$ is a value of light-adapted steady state fluorescence. Fv/Fm is a value of PSII maximum quantum yield after dark adaptation and is an informative criteria to study induced changes in PSII (Sanusi et al., 2011). Y(II) is a value of PSII effective quantum yield. $\mathrm{qP}$ is a coefficient of photochemical quenching, $\mathrm{qN}$ is a coefficient of nonphotochemical quenching. The NPQ parameter reflects the measure of nonphotochemical fluorescence quenching. Y(NPQ) is the quantum yield of regulated energy dissipation in PS II and $\mathrm{Y}(\mathrm{NO})$ is the quantum yield of nonregulated energy dissipation in PS II, were calculated according (Kramer et al., 2004).

\section{Measurements of P700 Redox State}

The P700 redox state was measured by Dual PAM100 with a dual wavelength (830/875 nm) unit, following the method of Klughammer and Schreiber (1994). Saturation pulses $\left(10,000 \mu \mathrm{mol}\right.$ photons $\left.\mathrm{m}^{-2} \mathrm{~s}^{-1}\right)$, were used for assessment of $\mathrm{P} 700$ parameters. The $\mathrm{P} 700^{+}$ signals (P) may vary between a minimal (P700 fully reduced) and a maximal level (P700 fully oxidized). The P700 fully oxidized level-Pm value, was determined using a saturation pulse after pre-illumination with a farred light. P'm was determined similarly to Pm, but using the actinic light background instead of a far-red illumination. $\mathrm{Y}(\mathrm{I})$ is the PSI photochemical quantum yield, it was measured by a fraction of overall P700 that in a given state was reduced and not limited by the acceptor side. It was calculated as $\mathrm{Y}(\mathrm{I})=\left(\mathrm{P}^{\prime} \mathrm{m}-\mathrm{P}\right) / \mathrm{Pm}$. $\mathrm{Y}(\mathrm{ND})$ value represents a fraction of overall P700 that is oxidized in a given state. Y(ND) value may be enhanced by a transthylakoid proton gradient (photosynthetic control at cyt $\mathrm{b} / \mathrm{f}$ complex as well as down-regulation of PSII) and PSII photodamage. It was calculated as Y(ND) $=\mathrm{P} / \mathrm{Pm}$. $\mathrm{Y}(\mathrm{NA})$ value represents a fraction of overall P700 that cannot be oxidized by a saturation pulse in a given state due to a lack of oxidized acceptors. It was calculated as $\mathrm{Y}(\mathrm{NA})=P m-\mathrm{P}^{\prime} \mathrm{m} / P m$.

\section{Measurement of PS I and PS II Electron Flows}

PSI and PSII electron flows were calculated as follows: $\operatorname{ETR}(\mathrm{I})=\mathrm{Y}(\mathrm{I}) \times \mathrm{PAR} \times \alpha \mathrm{I}, \operatorname{ETR}(\mathrm{II})=\mathrm{Y}(\mathrm{II}) \times$ PAR $\times \alpha$ II (Miyake et al., 2005). $\alpha$ I and $\alpha$ II were obtained as $\alpha \mathrm{I}=p \times d \mathrm{I}$ and $\alpha \mathrm{II}=\mathrm{p} \times d \mathrm{II}$, where $p$ is the absorptance (the fraction of the incident light absorbed by leaves) and $d \mathrm{I}$ and $d \mathrm{II}$ are fractions of the absorbed light distributed to PSI and PSII, respectively.

\section{Data Processing and Analysis}

All measurements were carried out in at least 6 repeats. Here the mean values and standard errors are 
presented. The Student's t-test $(\alpha=0.05)$ was used for statistical analysis of significant differences.

\section{Results}

The basic fluorescence parameters measured in the en:chlorina-7 mutant were compared with the line 3629 (as a wild type (WT)) and are presented in Table 1.

\section{Mean Values \pm Standard Error}

Most fluorescence parameters differed strongly between en:chlorine-7 and line 3629, among them the PSI parameter - Pm. The mutant plants had much lower value of $P m(35 \%)$ as compared with a WT. The Pm value depends on a maximum amount of the photooxidizable P700, which is a parameter representing an amount of efficient PSI complexes (Huang et al., 2010). To visualize the P700 signal changes of the original kinetics as well as chlorophyll fluorescence kinetics are presented in Fig. 1.

For comparison, Fm - one of the main PSII fluorescence parameters was reduced only on $10 \%$ in mutant plants. These changes indicate a greater reduction of PSI complexes as compared with PSII complexes.

However some changes in values of fluorescence parameters pointed at the PSII diminution. Fo parameter was higher in the chlorina mutant line and, as a consequence, mutant plants had reduced effective quantum yield of PSII photochemistry. An increase of Fo directly have influenced on the maximum quantum yield of PSII after dark adaptation $(\mathrm{Fv} / \mathrm{Fm})$. The rate of electron transport was also lower in mutant plants. Especially clearly a decrease of PSII efficiency was seen in the analysis of ETR(II) light curves (Fig. 2).
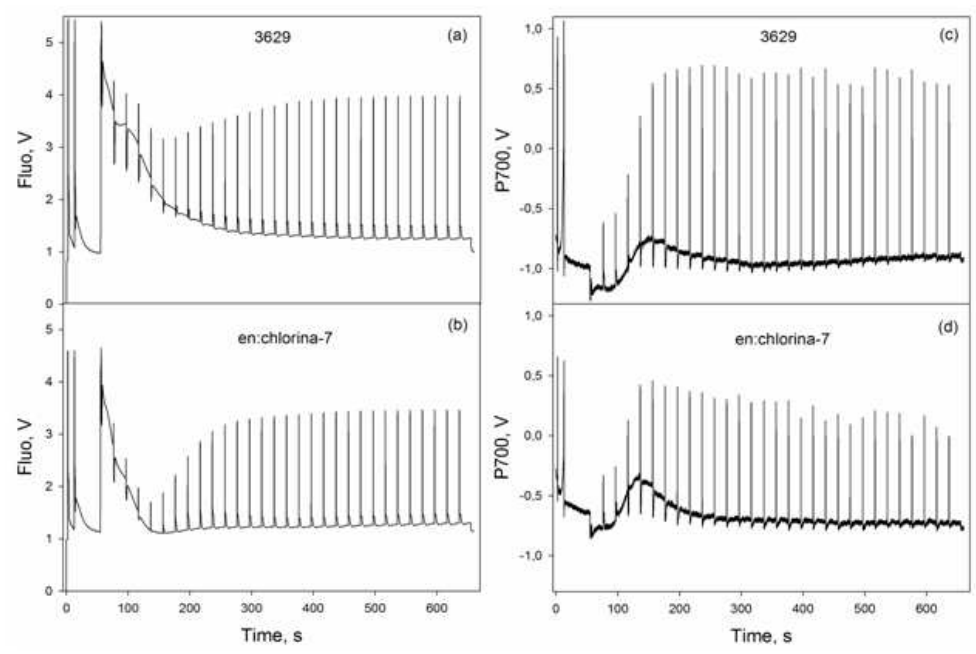

Fig. 1. Slow kinetics of chlorophyll $a$ fluorescence induction $(\mathrm{a}, \mathrm{b})$ and kinetics of the changes of P700 signal (c, d) for the line 3629 $(\mathrm{a}, \mathrm{c})$ and for the mutant line en:chlorina-7 (b, d)

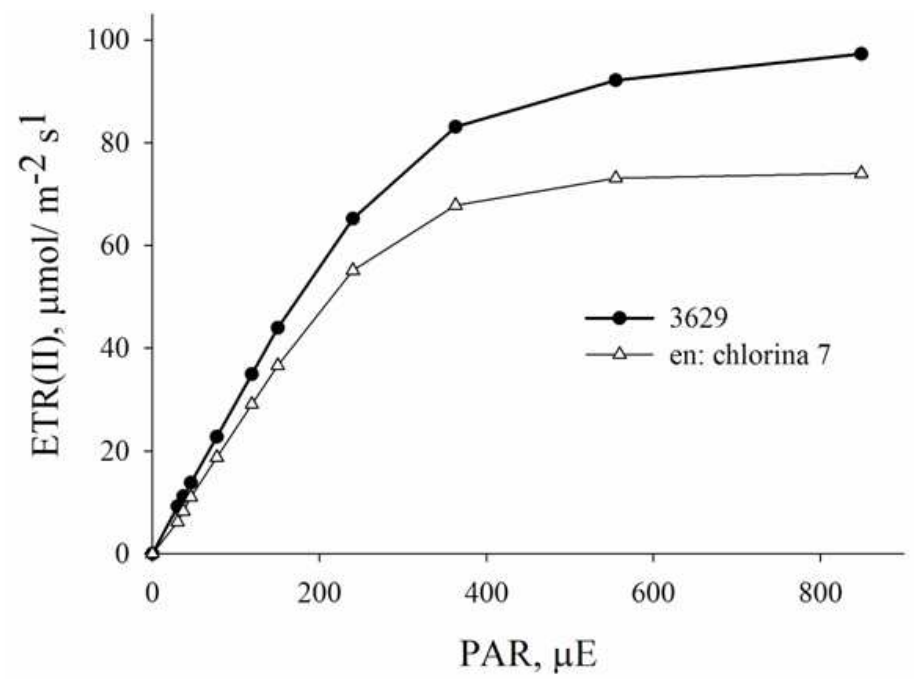

Fig. 2. Light curves, reflecting dependence of ETRII on the intensity of an actinic light in the line 3629 and in the mutant line en:chlorina-7 
Table 1. Values of chlorophyll fluorescence and P700 parameters measured in the line 3629 (WT) and in the mutant line en:chlorine-7

\begin{tabular}{lll}
\hline Parameters & Line 3629 & en:chlorine-7 \\
\hline Fo & $0.83 \pm 0.03$ & $1.00 \pm 0.01$ \\
Fm & $5.34 \pm 0.14$ & $4.69 \pm 0.14$ \\
Fv/Fm & $0.84 \pm 0.00$ & $0.79 \pm 0.00$ \\
Y(II) & $0.69 \pm 0.01$ & $0.64 \pm 0.02$ \\
ETR(II) & $43.75 \pm 0.92$ & $40.10 \pm 1.13$ \\
qP & $0.87 \pm 0.02$ & $0.87 \pm 0.02$ \\
qN & $0.31 \pm 0.00$ & $0.29 \pm 0.00$ \\
NPQ & $0.36 \pm 0.00$ & $0.32 \pm 0.00$ \\
Y(NPQ) & $0.08 \pm 0.00$ & $0.08 \pm 0.00$ \\
Y(NO) & $0.22 \pm 0.01$ & $0.27 \pm 0.01$ \\
Pm & $2.12 \pm 0.01$ & $1.36 \pm 0.15$ \\
Y(I) & $0.78 \pm 0.05$ & $0.86 \pm 0.00$ \\
ETR(I) & $48.95 \pm 2.90$ & $54.25 \pm 0.21$ \\
Y(ND) & $0.02 \pm 0.00$ & $0.03 \pm 0.01$ \\
Y(NA) & $0.21 \pm 0.05$ & $0.11 \pm 0.00$ \\
\hline
\end{tabular}

At low intensities of actinic light the difference between ETR(II) of the en:chlorina-7 and the WT was relatively small (5-10\%), but at high intensities of actinic light ETR(II) showed a great difference (up to $30 \%$ ). The analysis of light curves has not revealed so a big difference between the en:chlorina-7 and the WT in any other parameter.

It is interesting to note that, there was no significant difference in coefficients of photochemical quenching (qP) and nonphotochemical quenching $(\mathrm{qN})$ between the mutant and the WT. However the value of NPQ ratio was higher in the WT compared to the yellow-green mutant. The NPQ value reflects PS II down-regulation, which is a protective mechanism against excess light intensity and also the NPQ value could be an indicator of transthylakoid proton gradient (Busch et al., 2008). We expected some differences in NPQ values between the mutant and the WT at the higher intensity of actinic light, but there were no significant changes in light curves. Values of quantum yields of regulated (Y (NPQ)) and non-regulated (Y (NO)) energy dissipation were slightly increased in mutant plants. Nevertheless, such variations are insufficient for making assumptions about changes in the energy dissipation of PSII.

An opposite pattern is observed in values of complimentary quantum yields of PSI photochemistry (Table 1). Much lower values of Y(NA) in the chlorina mutant indicated a decreased acceptor side limitation of PSI. Consequently, an increased Y(ND) parameter, indicated a higher oxidation status (redox poise) of P700 (donor side limitation of PSI) in the mutant compared to the WT. However, a reduction of $\mathrm{Y}$ (NA) in the en:chlorine-7 may be caused by an activation of Calvin cycle, which in turn increases the quantum yield of PSI photochemistry (Y(I)) and thus, the more effective rate of electron transport (ETR (I)) is observed.

\section{Discussion}

Previous studies have revealed non-synonymous mutations in the en: chlorine 7 plastid genes - psaA, psbB (Markin et al., 2016). Hence the chlorophyll deficient phenotype may be associated with an impaired function of core subunits of PSI ( $p s a A$ ) either PSII $(p s b B)$ or with the disturbance of both proteins. It is important to note that, if these mutations had led to complete loss of proteins function, a white phenotype in plants would have been detected, similar to that have been observed in tobacco deletion mutants (Leelavathi et al., 2011). Lack of published information about chlorina mutants with extranuclear genetic origin and a rarity of studies of mutation in psaA and $p s b B$ in higher plants, it makes it difficult to approve some statements, but the assumption could be made.

According to data obtained from PAM fluorometer measures a few assumptions could be established. The first one is that, there is a depletion of P700 in mutant plants. This is evidenced by a considerable decrease of the $P m$ value, which is the direct indicator of a low content of photo-oxidizable PSI (Grieco et al., 2012). A possible reason for such significant reduction of PSI could be psaA mutation - Thr528Ile. However to confirm this assumption further investigations, especially proteomic analyses, are required.

Another assumption could be made, that the mutation in $p s b B$ (His 157Tyr) associated with a reduction of PSII. In the en:chlorina-7 line the Fm value was not decreased so greatly as the $P m$ value, although mutant plants had worsening in large number of PSII parameters-Fo, Fv/Fm, Y(II), ETR(II).

Thus we assume that both mutations have phenotypic effect, however which genetic changes lead to chlorophyll deficient phenotype is still not entirely clear. On the one hand the lower chlorophyll content may be observed due to the reaction centers reduction. The decrease of $P m$ and Fm values may be associated with RC lessening of PSI and PSII, respectively, but without additional molecular analyses, we are not able to exactly establish RC lessening. It is interesting to note that Brestic et al. (2015) also revealed a great decrease of the $P m$ value in wheat yellow-green mutant lines.

On the other hand the reason of chlorophyll deficiency may be due to a diminution of light harvesting complexes. This assumption can be supported by an alb chlorophyll ratio increase in en:chlorina-7 plants. In spite the fact that the Chl-b breakdown is observed at degradation of LHC, nevertheless the alb chlorophyll ratio is not a sufficient criteria for unambiguous conclusion (Kovács et al., 2006; Tanaka and 
Tanaka, 2011). More informative for the confirmation of the decreasing LHC content are ETR(II) changes. Mutant plants had the reduced rate of electron transport and without the reduction of PSII antenna there must be an excess of excited electrons. The excess of excited electrons had to impact on parameters of an energy dissipation, but values of energy dissipation were similar in both mutant and WT plants even in high actinic light conditions.

Although we tend to think that a decline of chlorophyll content in the en:chlorina-7 is primarily associated with a decrease in LHC, there is an insufficient evidence for the definite approval. We may expect that further studies using yellow-green mutants may resolve this dispute. As well as the conducted research contributes to knowledge in a field of chlorophyll deficient phenotype development reasons.

\section{Conclusion}

The conducted research revealed a low content of photo-oxidizable PSI and an impaired quantum yield of PSII photochemistry in the en:chlorina-7 line as compared with the wild type. These disturbances of PSI and PSII in the yellow-green plastome mutant line of sunflower (en:chlorina-7) are associated with mutations in $p s a A$ and $p s b B$, respectively. The results obtained could be useful for investigations in a field of the genetics of photosynthesis.

\section{Acknowledgement}

This research was supported by Ministry of Education and Science of Russian Federation, project no. 40.91.2014/K.

\section{Author's Contributions}

M.S. Makarenko: Participated in data analysis and the entire process of the article preparation

N.V. Kozel: Participated in all laboratory trials and writing manuscript chapter - «Materials and Methods»

A.V. Usatov: Designed the research plan and organized the investigation

O.F. Gorbachenko: Participated in plant material preparation and some experiments

N.G. Averina: Coordinated the data-analysis

\section{Ethics}

This article is original and contains unpublished material. The authors declare that there is no conflict of interest regarding publication of this paper. The authors declare that no ethical issues are going to arise after the work has been published.

\section{References}

Asakura, Y., S. Kikuchi and M. Nakai, 2008. Nonidentical contributions of two membrane-bound cpSRP components, cpFtsY and Alb3, to thylakoid biogenesis. Plant J., 56: 1007-1017.

DOI: 10.1111/j.1365-313X.2008.03659.X

Beletskii, Y.D., E.K. Razoriteleva and Y.A. Zhdanov, 1969. Cytoplasmic mutations of sunflower induced by n-nitrosomethylurea. Dokl. Akad. Nauk, 186: 1425-1426.

Brestic, M., M. Zivcak, K. Kunderlikova, O. Sytar and H. Shao et al., 2015. Low PSI content limits the photoprotection of PSI and PSII in early growth stages of chlorophyll b-deficient wheat mutant lines. Photosynthesis Res., 125: 151-166. DOI: $10.1007 / \mathrm{s} 11120-015-0093-1$

Busch, F., N. P.A. Huner, I. Ensminger, 2008. Increased Air Temperature during Simulated Autumn Conditions Impairs Photosynthetic Electron Transport between Photosystem II and Photosystem I. Plant Physiol., 147: 402-414. DOI: $10.1104 / p p .108 .117598$

Chen, H., Z. Cheng, X. Ma, H. Wu and Y. Liu et al., 2013. A knockdown mutation of YELLOW-GREEN $L E A F_{2}$ blocks chlorophyll biosynthesis in rice. Plant Cell Reports, 32: 1855-1867. DOI: $10.1007 / \mathrm{s} 00299-013-1498-y$

Grieco, M., M. Tikkanen, V. Paakkarinen, S. Kangasjärvi and E.M. Aro et al., 2012. Steady-state phosphorylation of light-harvesting complex II proteins preserves Photosystem I under fluctuating white light. Plant Physiol., 160: 1896-1910. DOI: $10.1104 /$ pp.112.206466

Harper, A.L., S.E. von Gesjen, A.S. Linford, M.P. Peterson and R.S. Faircloth et al., 2004. Chlorophyllide $a$ oxygenase mRNA and protein levels correlate with the chlorophyll $a / b$ ratio in Arabidopsis thaliana. Photosynthesis Res., 79: 149-159. DOI: 10.1023/B:PRES.0000015375.40167.76

Huang, W., S.B. Zhang and K.F. Cao, 2010. Stimulation of cyclic electron flow during recovery after chilling-induced photoinhibition of PSII. Plant Cell Physiol., 51: 1922-1928. DOI: 10.1093/pcp/pcq144

Klughammer, C. and U. Schreiber, 1994. An improved method, using saturating light pulses, for the determination of photosystem I quantum yield via P700\&\#x002B; -absorbance changes at $830 \mathrm{~nm}$. Planta, 192: 261-268. DOI: 10.1007/BF01089043

Kovács, L., J. Damkjær, S. Kereïche, C. Ilioaia and A.V. Ruban et al., 2006. Lack of the light-harvesting complex $\mathrm{CP}_{24}$ affects the structure and function of the grana membranes of higher plant chloroplasts. Plant Cell, 18: 3106-3120.

DOI: $10.1105 / \mathrm{tpc} .106 .045641$ 
Kramer, D.M, G. Johnson, O. Kiirats and G.E. Edwards, 2004. New fluorescence parameters for the determination of $\mathrm{Q}_{\mathrm{A}}$ redox state and excitation energy fluxes. Photosynthesis Res., 79: 209-218. DOI: 10.1023/B:PRES.0000015391.99477.0d

Leelavathi, S., A. Bhardwaj, S. Kumar, A. Dass and R. Pathak et al., 2011. Genome-wide transcriptome and proteome analyses of tobacco $p s a A$ and $p s b A$ deletion mutants. Plant Molecular Biol., 76: 407-423. DOI: $10.1007 / \mathrm{s} 11103-011-9731-\mathrm{y}$

Li, N., J. Jia, C. Xia, X. Liu and X. Kong, 2013. Characterization and mapping of novel chlorophyll deficient mutant genes in durum wheat. Breed. Sci., 63: 169-175. DOI: 10.1270/jsbbs.63.169

Lysenko, V.S., T.V. Varduny, E.I. Simonovich and O.I. Chugueva, 2014. Far-red spectrum of second emerson effect: A study using dual-wavelength pulse amplitude modulation fluorometry. Am. J. Biochem. Biotechnol., 10: 234-240.

DOI: 10.3844/ajbbsp.2014.234.240

Markin, N., A. Usatov, M. Logacheva and V. Vasilenko, 2016. Variability of chloroplast DNA of extranuclear sunflower mutants. Am. J. Biochem. Biotechnol., 12: 72-78. DOI: 10.3844/ajbbsp.2016.72.78

Miyake, C., M. Miyata, Y. Shinzaki and K. Tomizawa, 2005. $\mathrm{CO}_{2}$ response of cyclic electron flow around PSI (CEF-PSI) in tobacco leavesrelative electron fluxes through PSI and PSII determine the magnitude of non-photochemical quenching (NPQ) of chl fluorescence. Plant Cell Physiol., 46: 629-637. DOI: 10.1093/pcp/pci067

Oxborough, K. and N.R. Baker, 1997. Resolving chlorophyll a fluorescence images of photosynthetic efficiency into photochemical and nonphotochemical components - calculation of qP and Fv-/Fm-; without measuring Fo-. Photosynthesis Res., 54: 135-142. DOI: 10.1023/A: 1005936823310
Preiss, S. and J.P. Thornber, 1995. Stability of the apoproteins of light-harvesting complex I and II during biogenesis of thylakoids in the chlorophyll bless barley mutant chlorina f2. Plant Physiol., 107: 709-717. DOI: $10.1104 /$ pp.107.3.709

Rassadina, V.V., A.V. Usatov, G.M. Fedorenko and N.G. Averina, 2005. Activity of the system for chlorophyll biosynthesis and structural and functional organization of chloroplasts in a Plastome en:chlorina-5 sunflower mutant. Russian J. Plant Physiol., 52: 606-615. DOI: $10.1007 / \mathrm{s} 11183-005-0090-\mathrm{x}$

Sanusi, R.A.M., A.A. Nuruddin and H.A. Hamid, 2011. Leaf chlorophyll fluorescence and gas exchange response to different light levels in Platycerium bifurcatum. Am. J. Agric. Biol. Sci., 6: 214-220. DOI: 10.3844/ajabssp.2011.214.220

Tanaka, R. and A. Tanaka, 2011. Chlorophyll cycle regulates the construction and destruction of the light-harvesting complexes. Biochimica et Biophysica Acta, 1807: 968-976. DOI: 10.1016/j.bbabio.2011.01.002

Usatov, A.V., E.K. Razoriteleva, E.V. Mashkina and I.I. Ulitcheva, 2004. Spontaneous and nitrosomethylurea-induced reversions in plastome chlorophyll mutants of sunflower Helianthus annuus L. Russian J. Genet., 40: 186-192. DOI: 10.1023/B:RUGE.0000016993.37051.ef

Wang, L., C. Yue, H. Cao, Y. Zhou and J. Zeng et al., 2014. Biochemical and transcriptome analyses of a novel chlorophyll-deficient chlorina tea plant cultivar. BMC Plant Biol., 14: 352-352.

DOI: $10.1186 / \mathrm{s} 12870-014-0352-\mathrm{x}$ 